\title{
LA CONSTRUCCIÓN DE FRONTERAS COMO UN PROCESO BIDIRECCIONAL. EL CASO DE SudÁFrica
}

\author{
Adela Beatriz Escobar Cristiani* \\ María del Rayo Ventura Navarrete***
}

\section{Resumen}

Tradicionalmente, las fronteras se conciben como "líneas en el mapa", elementos geográficos que delimitan a los Estados de manera más o menos clara y estable. En esta lógica, se considera que las fronteras separan las identidades sociales y acotan la actividad de los actores involucrados, en un proceso vertical que va de arriba (el Estado) hacia abajo (la población). Sin embargo, un examen más cuidadoso revela que esta concepción es insuficiente para entender la dinámica del mundo actual. En los últimos años se ha extendido la percepción de las fronteras como elementos que se construyen a partir de prácticas sociales más amplias, que no solo estarán determinadas por el Estado, sino que involucran a múltiples actores sociales a través de diversas formas. El artículo explora esta concepción de las fronteras como elementos en continua construcción en un proceso bidireccional, tomando como punto de referencia el caso de Sudáfrica.

Palabras clave: Sudáfrica, fronteras, migrantes, Estado, ONG, actores sociales.

\section{BORDER BUILDING AS A BIDIRECTIONAL PROCESS. THE CASE OF SOUTH AFRICA}

\section{Abstract}

Traditionally, borders are conceived as "lines on the map", geographic elements that

* Doctora en Estudios de Asia y África especialidad África, El Colegio de México (México). Profesora de la Facultad de Ciencias Políticas y Sociales de Ta Universidad Nacional Autónoma de México (México). [abescobar@colmex.mx].

** Doctora en Estudios de Asia y África especialidad África, El Colegio de México (México). Investigadora independiente residente en Sudáfrica. [venturama@yahoo.com].

Recibido: 1 de marzo de 2018 / Modificado: 28 de mayo de 2018 / Aceptado: 27 de julio de 2018.

Para citar este artículo

Escobar Cristiani, A. B. y Ventura Navarrete, M. R. (2018). La construcción de fronteras como un proceso bidireccional. El caso de Sudáfrica. OPERA, 23, 115-138.

DoI: https://doi.org/10.18601/16578651.n23.08 
delimit states more or less clearly and stably. In this logic, it is considered that borders separate social identities and delimit the activity of social actors, in a vertical process that goes from above (the State) downwards (the population). However, a more careful examination reveals that this conception is insufficient to understand the dynamics of the current world. In recent years, it has been pointed out that it is more appropriate to understand borders as elements that are constructed from broader social practices that will not only be determined by the State, but involve numerous social actors through multiple ways. The article explores this conception of borders as elements in continuous construction, through the study of the South African case.

Key words: South Africa, borders, migrants, state, NGO, social actors.

\section{INTRODUCCIÓN}

En las últimas décadas, las fronteras han sido objeto de intensa reflexión, a causa de diversos factores que han ido modificando nuestra comprensión de la realidad internacional: la caída del Muro de Berlín y el fin de la Guerra Fría; el fortalecimiento de la Unión Europea y su posterior cuestionamiento con la decisión británica de abandonarla; el desarrollo de tecnologías de la información que han generado nuevas formas de comunicación y cambios en los modos de contacto económico; los procesos de globalización; la creciente importancia de las migraciones internacionales; el ascenso de movimientos relacionados con la identidad alrededor del planeta, así como la extensión de movimientos políticos y sociales de diversa índole que operan por encima de las fronteras (Newman y Paasi, 1998; Beurskens y Miggelbrink, 2017; Parker y Vaughan-Williams et al., 2009).

Todos estos cambios han provocado que los significados de las fronteras se alteren, y que se debatan sus funcionalidades y su relación con distintos temas, incluyendo la soberanía, la seguridad, el control de la migración, el acceso a recursos y a derechos, entre muchos otros (Beurskens y Miggelbrink, 2017; Aquino y Varela, 2012).

La visión más tradicional considera a las fronteras como elementos geográficos claramente establecidos que separan a unidades políticas bien delimitadas. Sin embargo, las nuevas perspectivas muestran que se trata en realidad de algo mucho más complejo. Al circunscribir de manera espacial a los países, las fronteras establecen en principio sitios de identidad. No solo dividen unidades políticas, sino sobre todo muestran la separación entre un "nosotros" definido socialmente y un "otro" del que nos distinguimos. Asimismo, las fronteras establecen espacios de institucionalización y socialización. Más allá de delimitar el territorio, designan también a quienes se ubican bajo la autoridad de un Estado y a quienes son beneficiarios de los derechos que este reconoce (Foucher, 1991).

Desde el punto de vista geopolítico, las fronteras también tienen un profundo significado simbólico que supera los espacios físicos concretos. Así, por ejemplo, las fronteras que dividían a Europa durante la Guerra Fría fraccionaban al mundo de una manera más profunda, pero menos tangible, pues marcaban la 
separación entre el socialismo y el capitalismo (Rumford, 2012). En el mismo sentido, las fronteras europeas y estadounidenses marcan en la actualidad, no únicamente un punto específico donde se obstaculiza la movilidad de los migrantes provenientes de países de África, Asia y América Latina, sino que representan la división más honda entre los llamados Norte y Sur globales.

El artículo explora esta concepción más amplia de frontera, tomando a Sudáfrica como referente. Se estudia primero la definición estatocéntrica clásica de las fronteras, basada en la concepción westfaliana del mundo que históricamente ha dominado el análisis de la realidad internacional. A continuación, se revisa el concepto desde un punto de vista no estatocéntrico, como algo que se construye a través de la operación continua de múltiples actores. Finalmente, se revisa el caso de Sudáfrica para comprender en qué medida es posible aplicar cada una de estas visiones.

\section{UNA VISIÓN EMBRIONARIA: LAS FRONTERAS COMO LÍNEAS QUE DIVIDEN}

En el sentido más tradicional, las fronteras son líneas que señalan los bordes del territorio de un país y que lo separan de otras unidades del mismo tipo (Minghi, 1991). Por tanto, históricamente se les ha concebido como "entidades empíricas" que dividen de manera tangible el espacio mundial, para formar unidades fijas que cambian casi exclusivamente como resultado de conflictos (Paasi, 1998). Relacionada de manera directa con la concepción del mundo que derivó de la llamada Paz de Westfalia
(Beurskens y Miggelbrink, 2017), esta forma de entender las fronteras ha sido consagrada por el derecho internacional.

La perspectiva westfaliana es básicamente estatocéntrica, pues considera que las soberanías estatales delimitadas por las fronteras constituyen la vía de realización de un destino histórico (Paasi, 1998). Tal idea deriva en lo que algunos autores denominan "la trampa territorial" (Paasi, 2005), una forma de entender la realidad que concibe al Estado como "el embalaje geográfico" de la sociedad. Siguiendo esta lógica, se estima que el Estado moderno requiere espacios territoriales claramente delimitados. Por tanto, se supone que el propio Estado tendrá el papel central en la creación y la conservación de las fronteras, a través de la afirmación de su autoridad y soberanía en el territorio que estas demarcan.

El tema de la autoridad y la soberanía se vuelve especialmente delicado dentro de esta perspectiva tradicional porque se suele considerar a las zonas fronterizas como áreas problemáticas en dos sentidos. En primer lugar, es común entenderlas como un espacio indómito e inestable. Por esta razón, una de las grandes preocupaciones consiste en encontrar las vías adecuadas para que sean domesticadas e integradas a la órbita de organización y civilización de la soberanía estatal (Newman y Paasi, 1998). En segundo lugar, el espacio más allá de las fronteras se percibe como un ámbito de riesgo constante donde se ubican distintas amenazas potenciales para el Estado-nación (Rioja, 2015).

Muchos de esos riesgos, tal como se les concibe desde un punto de vista tradicional, se relacionan con la "violación" de las fronteras. 
Se parte de la idea de que, al quedar trazada, la línea fronteriza debe constituir una separación rotunda, por lo cual nadie debe cruzarla (Parker y Vaughan-Williams et. al., 2009) sin la debida autorización otorgada por el Estado. Hacer lo contrario, en forma individual o grupal, constituiría una agresión.

Para cumplir con su destino histórico por medio de la solución de los múltiples retos que suponen las fronteras, el Estado debe realizar una serie de tareas tendientes a asegurar el funcionamiento interno del área bajo su control y a evitar riesgos procedentes del exterior. De esta manera, el Estado aparece como la entidad encargada de enfrentar las amenazas, no solo internas, sino también externas, a fin de garantizar su propia supervivencia (Rioja, 2015). A nivel interno debe asegurar, por ejemplo, que la población se identifique con los espacios y simbolismos demarcados con la frontera (Paasi, 2005), a través de una serie de prácticas, instituciones y narrativas. A nivel externo, debe "cerrar" las fronteras en contra de los riesgos provenientes de otros países.

Respecto del control de las amenazas, la seguridad de las fronteras ha sido asociada de forma directa "al factor político-militar del control interno y su proyección externa"(Rioja, 2015, p. 31). Se considera que el Estado debe consolidar las barreras tanto físicas (cercas, muros, barreras vehiculares, presencia de personal de seguridad) como intangibles (por ejemplo, la legislación) para impedir el tránsito de posibles amenazas (drogas, armas, terroristas) a través de sus fronteras.

En este modelo, la regulación de la movilidad adquiere una importancia particular (Pfoser, 2015). Como se indicó, dado que el espacio más allá de las fronteras se concibe como fuente de amenazas potenciales, la "violación" de la frontera se considera una agresión contra el Estado. Por tal motivo, el cruce no autorizado se verá como algo inaceptable y se ubicará fuera del marco legal. Si bien en principio esas medidas se suponen destinadas a impedir el cruce de amenazas, también colocarán en un estatus de ilegalidad a los migrantes no autorizados.

El control de la migración no autorizada aparece entonces como parte de un conglomerado más amplio, junto con el combate a la delincuencia y la lucha contra el terrorismo. De forma velada, al migrante se le incorpora en una misma categoría con el delincuente y el terrorista (Aquino y Varela, 2012). Como resultado, el Estado consagra la tarea de "controlar" al migrante como fundamental para garantizar su propia seguridad.

Sin embargo, Aquino y Varela (2012) explican cómo el control del migrante en realidad adquiere otras connotaciones. La condición de ilegalidad de quienes cruzan la frontera de forma no autorizada es, más que un fenómeno natural provocado por el cruce mismo, un "estado producido por la propia ley". Es decir, se trata de un producto de la socialización que, de acuerdo con estos autores, buscaría expresamente garantizar la existencia de "mano de obra flexible, disciplinada y precaria" a través de la exclusión tanto social como política (p. 8).

Este proceso de exclusión es posible únicamente con el apoyo de narrativas que aludan a la identidad y las fronteras. Como señalan Newman y Paasi (1998), las narrativas se manifiestan en las prácticas culturales más exten- 
didas. Constituyen además un elemento de control porque su construcción se encuentra mediada por diversas instituciones sociales y políticas. A través de ellas las sociedades dan sentido y ordenan simbólicamente el espacio delimitado por la frontera. Por ello, en última instancia, se relacionarán de manera directa con la distribución del poder en una sociedad determinada. En consecuencia, para entender las fronteras, se vuelve necesario saber quién domina las narrativas, quién es incluido o excluido en ellas, y cómo se producen y reproducen las imágenes del nosotros y el otro (Newman y Paasi, 1998). Incluso los sistemas legales reflejan en buena medida estas narrativas de control, tal y como se observa en el caso de la migración.

En síntesis, desde la perspectiva estatocéntrica, los Estados tienen un papel decisivo en la producción y reproducción de las fronteras, especialmente "a través de la socialización espacial y la territorialización de significado, que ocurren en muchas formas a través de la educación, la política, la administración y el Gobierno" (Paasi, 1998, p. 69).

Sin embargo, en los últimos años se han hecho evidentes las limitaciones de esa visión de las fronteras, pues deriva en una comprensión restringida de su funcionamiento y sus significados (Pfoser, 2015). Un primer aspecto problemático consiste en que se naturalizan muchos elementos que en realidad se construyen socialmente. Y por esta misma razón, no se explican las relaciones de poder involucradas en la construcción de las fronteras (Paasi, 2005), ni sus significados en la "organización y reproducción de la vida social, la territorialidad y el poder" (Paasi, 1998, p. 69).

\section{HACIA UNA NUEVA COMPRENSIÓN DE LAS FRONTERAS}

El contexto actual demanda un enfoque crítico respecto del estudio de las fronteras. Estas aparecen ahora como realidades cambiantes, de modo que la visión estatocéntrica se vuelve insuficiente para dar cuenta de la participación de otros actores que generan distintos tipos de interacciones (Brunet-Jailly, 2005). A partir de estos elementos, se cuestiona la idea de "las fronteras estatales" como una realidad unificada o fija, y se hace necesario pensar desde nuevas perspectivas quién actúa en las fronteras y cómo lo hace.

En este replanteamiento conceptual, el punto de partida lo constituye el cuestionamiento de la visión de las fronteras como un "espacio naturalizado"1. En cambio, se considera la "fronterización" como un proceso de construcción continua (Newman y Paasi, 1998). En última instancia, la frontera se ubica como un medio para las acciones humanas, no como un fin en sí mismo. La gente participa en su construcción, al igual que en la construcción del territorio (Pfoser, 2015), en

1 Sin embargo, señalar la no naturalización de las fronteras no equivale a sostener que las mismas carezcan de valor. A pesar de su carácter más o menos arbitrario, las fronteras tienen profundos significados simbólicos, culturales, históricos y religiosos para las comunidades sociales (Newman y Paasi, 1998). A menudo, el propio debate acerca de su significado es un indicio de su importancia simbólica. 
un proceso que cuestiona de manera constante los espacios simbólicos de control establecidos por el Estado. Así, la frontera adquiere múltiples significados, a causa de la variedad de contenidos políticos, geográficos y simbólicos que pone en juego.

En este sentido, Rumford (2012) considera que en un contexto contemporáneo es especialmente interesante estudiar los procesos de construcción de las fronteras a cargo de diversos actores que participan de nuevas formas y en una variedad de locaciones. De hecho, las fronteras dejan de verse como algo que limita espacios físicos contiguos, para entenderse como algo que puede separar, incluso, a entidades alejadas geográficamente.

Por su parte, Werlen (2005) señala las ventajas de analizar las condiciones sociales, las actividades y las relaciones socioespaciales que dan forma a las fronteras. A su vez, Zeller (2009) propone entender la configuración de la soberanía en el espacio que delimitan las fronteras como algo que se construye no solo de arriba hacia abajo (con el Estado como actor principal), sino también de abajo hacia arriba.

De esta manera, la frontera se explica tomando como eje, no el espacio por sí solo, sino sobre todo las acciones sociales que se generan en él. Esto nos permite entender la existencia de una multiplicidad de sujetos, tanto estatales como no estatales, internacionales, multinacionales o locales, que buscarán redefinir las relaciones entre los espacios físico y social (Newman y Paasi, 1998).

En el proceso confluye la acción de diversas instituciones que operan en un solo país (agencias gubernamentales, embajadas, consulados, las fuerzas armadas), instituciones formales que tienen presencia en varios países (como la Iglesia católica y otras instituciones religiosas globales, o las agencias especializadas de la Organización de las Naciones Unidas), así como actores no institucionales de la sociedad civil que incluyen activistas, productores agrícolas, comerciantes, empresarios, artistas, migrantes, entre otros (Portes, 2013). Cada uno de ellos deberá enfrentar un contexto particular (Werlen, 2005) y tendrá distinta capacidad de acción. Asimismo, desplegará intereses específicos que derivarán en acciones concretas. Igualmente, deberá desarrollar estrategias diversas en múltiples niveles, para aprovechar las oportunidades o hacer frente a la falta de ellas (Ley, 2005). Por tanto, cada actor involucrado tendrá percepciones diferentes de lo que significa la frontera, y cada uno tratará de utilizarla para sus propios fines, de manera tanto simbólica como práctica. Así, las poblaciones locales no necesariamente entenderán las fronteras de la misma forma que los Gobiernos (Rumford, 2012) o los agentes internacionales.

Por tal motivo, lo que ocurre en las fronteras puede revisarse desde distintas ópticas que tengan en cuenta las actividades políticas de los diferentes niveles del Gobierno, la actividad de las comunidades fronterizas y su cultura específica, así como las fuerzas del mercado y los flujos comerciales (Brunet-Jailly, 2005). Asimismo, como indican Newman y Paasi (1998), es importante considerar las formas de desarrollo a nivel regional, que influyen en y son influidas por la naturaleza de las relaciones transfronterizas. Todo ello permitiría comprender cómo interactúan estructura y agencia en la formación de las fronteras. 
En síntesis, una perspectiva no estatocéntrica debe considerar las prácticas y percepciones populares y de actores no estatales, enfocándose en la cultura y las identidades de la frontera. Ello permitirá entender algunas cuestiones centrales, como la relación que la gente establece con el Estado en esas zonas, o la forma en que las comunidades experimentan las fronteras, incluyendo si las perciben como algo flexible y en disputa o como algo no negociable (Pfoser, 2015). De esta manera, se pueden explicar mejor las relaciones dinámicas que se establecen entre las personas, los grupos locales y externos, y el Estado.

A continuación se analizará el proceso de construcción de las fronteras en el caso específico de Sudáfrica, desde un punto de vista histórico, a fin de comprenderlo como un fenómeno dinámico que se ha ido modificando a lo largo del tiempo.

\section{LA CONSTRUCCIÓN HISTÓRICA DE LAS FRONTERAS SUDAFRICANAS}

Es ampliamente conocido el hecho de que las fronteras actuales de los países africanos son sobre todo una creación colonial. Antes de la consolidación de la presencia europea en el continente, la concepción del espacio era en muchas regiones relativamente abierta, pues el movimiento de personas ocurría de acuerdo con las necesidades prácticas de cada momento.

A partir del siglo XviI, con la llegada de los primeros colonos europeos (sobre todo holandeses) encabezados por Jan van Riebeeck, al actual territorio de Sudáfrica, la concepción del espacio y las fronteras se fue modificando de manera progresiva. Van Riebeeck planteó como su objetivo extender el nombre de Cristo y promover los intereses de la Compañía Holandesa de las Indias Orientales (Vale, 2003). Esto significaba que el modelo inicial de colonización no se centraba todavía en un control absoluto del espacio ni en la demarcación de fronteras fijas claramente establecidas, sino que permitía (y de hecho buscaba) una mayor movilidad para los colonos. Sin embargo, se comenzaba a perfilar ya una preocupación por el control administrativo, misma que constituyó un antecedente directo de las políticas de control y construcción del Estado que se consolidarían más tarde.

Igualmente, se comenzó a presentar desde entonces una preocupación continua por establecer fronteras tanto territoriales como simbólicas entre la población de origen europeo y la africana. Vale (2003) considera que desde sus orígenes, el proceso de creación de fronteras en Sudáfrica no dependió de la idea de reciprocidad. A diferencia de lo que planteaba el modelo westfaliano, los europeos establecerían su dominio sobre los africanos y delimitarían las fronteras que los separaban sin que fuera necesario que las comunidades africanas otorgaran su reconocimiento. La consecuencia fue la creciente periferización de las poblaciones indígenas, que se fueron transformando en comunidades externas dentro de su propio territorio.

Los africanos no aceptaron la situación de manera pasiva. A medida que la presencia europea fue creciendo, se suscitaron conflictos relacionados con el establecimiento de fronteras entre las distintas comunidades. El control 
territorial europeo no se había consolidado de manera definitiva.

De hecho, todavía en las primeras décadas del siglo xix, la región de África Austral vivía importantes movimientos de población. El caso más notable es el de las migraciones ngoni, ocurridas en el periodo conocido como Mfecane, una etapa de cambio asociada con el ascenso del imperio zulú encabezado por Shaka a principios del siglo XIx (Saunders, 1983). En medio de la agitación social y de la derrota militar, diversos grupos emigraron hacia el norte. A principios de la década de 1840 , los ngoni alcanzaron el sur de la actual Tanzania. A partir de ahí, se subdividieron; algunos fueron hacia lo que hoy es Zambia y Malawi. Otros continuaron hacia el norte y llegaron incluso al Lago Tangañika (Kimambo, 1997). Se observa así que "en la mente de los pueblos indígenas la región estaba libre de fronteras" en el sentido westfaliano (Vale, 2003, p. 44), pues un movimiento de este tipo no podría entenderse en un contexto de fronteras fijas.

Sin embargo, a medida que el control ejercido por la población de origen europeo se fue consolidando, las fronteras fueron cerrándose cada vez más. De manera específica, el descubrimiento de diamantes y el desarrollo de la minería propiciaron un mayor interés por garantizar el control del territorio. La posterior realización de la Conferencia de Berlín, que configuró el mapa de la presencia europea en África, provocó una mayor estabilización de las fronteras.

A mediados del siglo $\mathrm{xx}$, la institucionalización del régimen del apartheid (vigente de 1948 a 1994) supuso el fortalecimiento de las fronteras externas, pero también de las fronteras ideológicas internas al consolidarse el proceso de periferización de las poblaciones africanas. Mediante la Promotion of Bantu Self Government Act de 1959, el Gobierno transformó las antiguas reservas creadas por la Land Act de 1913, en zonas separadas llamadas hogares nacionales (en inglés homelands) o bantustanes (Oxford, 2008). Se trataba de unidades que geográficamente se encontraban dentro del territorio sudafricano, pero eran consideradas, supuestamente, "independientes". Cada "hogar nacional" debía concentrar en su interior a la población de un grupo africano determinado. Al ser catalogadas como unidades "independientes", la población africana que residía en estos lugares recibía la ciudadanía de su bantustán, pero no la sudafricana, de manera que eran considerados "extranjeros" por las autoridades sudafricanas. Esto tenía un impacto en la movilidad de aquellos trabajadores que se dirigían a las ciudades, las minas y los campos de la población blanca, pues debían portar permanentemente una identificación denominada passbook, la cual incluía todos sus datos personales y su permiso de trabajo.

La frontera ideológica se extendió también con la creación de los townships, establecidos por el Gobierno sudafricano como zonas de residencia para la población no blanca en las afueras de las ciudades y los pueblos. En algunos periodos, el Gobierno buscó reforzar la división de los grupos poblacionales a través de leyes específicas como la Group Areas Act de 1950, la cual establecía que cada ciudad o pueblo debía separarse en zonas designadas para blancos, coloured, indios y africanos. Aquellas poblaciones que estuvieran viviendo en una parte considerada blanca según la nueva dis- 
posición, debían ser trasladadas a otra zona. En este marco, la Native Ressettlement Act, No. 19 de 1954 estableció que los africanos que vivieran en Johannesburgo serían removidos por la fuerza. Así, por ejemplo, el township conocido como Sophiatown fue destruido y sus pobladores se incorporaron a otro township denominado Soweto (South African History On Line, s/f), situado al suroeste de Johannesburgo y famoso por haber sido el hogar de Nelson Mandela.

A pesar de todo, incluso en este contexto de crecientes restricciones, la movilidad conti- nuó como "una característica de la vida en África Austral" (Vale, 2003, p. 43). El movimiento de trabajadores hacia las minas y las granjas fue una constante que nutrió el engranaje del sistema económico sudafricano. Los migrantes podían ser sudafricanos de piel negra que eran obligados a desplazarse por medio de leyes coercitivas y segregacionistas, o bien, nacionales de países vecinos como Angola, Malawi, Swazilandia y Mozambique que eran llevados a Sudáfrica con el apoyo de instituciones de reclutamiento como la Cámara de Minas y, posteriormente, la Asociación del Witwatersrand

FIGURA 1. MAPA DE SUDÁFRICA

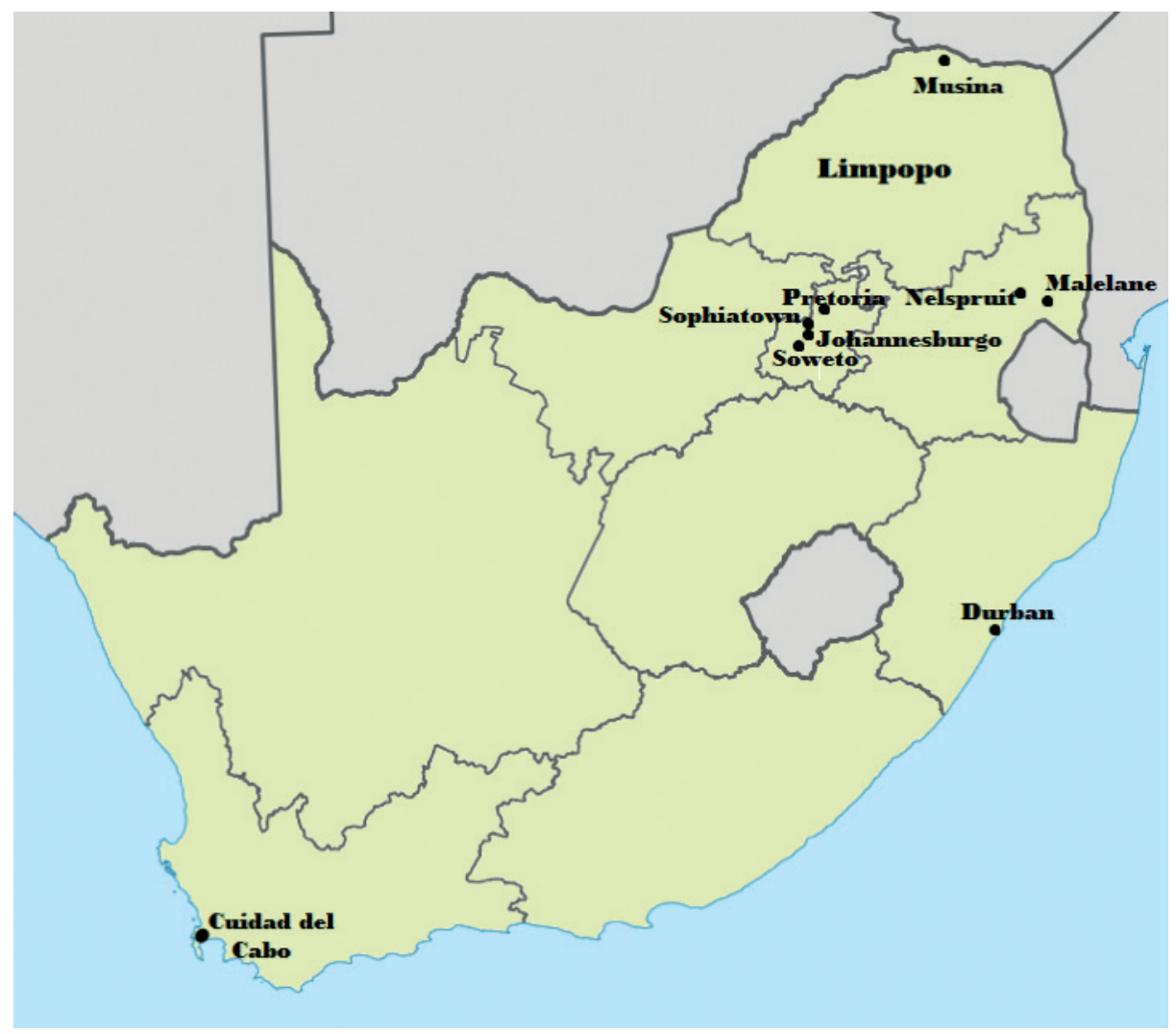

Fuente: modificado a partir de Wikipedia. 
para el Trabajo Indígena (Wenela) y la Oficina del Trabajo de África (тевA).

\section{NUEVAS TENDENCIAS DE MOVILIDAD EN LAS FRONTERAS}

La migración de personas hacia territorio sudafricano continuó en el periodo pos-apartheid, es decir, a partir de la llegada al poder del primer Gobierno democrático en 1994. No obstante, en esta nueva etapa histórica tuvieron lugar ciertas modificaciones importantes respecto al tránsito de personas a través de las fronteras. En primer lugar, los nuevos migrantes comenzaron a integrarse en actividades económicas distintas: además de trabajar en minas y campos, los migrantes incursionaron en el sector del comercio transfronterizo, que permite el intercambio de productos desde otros países hacia Sudáfrica, o en sentido contrario (Peberdy et al., 2015).

En segundo lugar, se registró un cambio en el origen geográfico de los migrantes. Crush, Chikanda y Skinner (2015) señalan que en el comercio a través de las fronteras participan ahora migrantes de países tan lejanos como Etiopía y Somalia. Con este nuevo perfil migratorio, y los esfuerzos gubernamentales por controlar ese tipo de migración, queda claro que, como se señaló en las páginas anteriores, las fronteras no necesariamente tienen una connotación geográfica de contigüidad. En realidad, la frontera no separa a Sudáfrica solo de sus vecinos inmediatos como Zimbabwe o Mozambique, sino que conceptualmente la separa del resto de África.

Muchos de los migrantes que vienen de estos países se dedican al comercio informal a través del establecimiento de pequeños locales comerciales o mesas improvisadas en las principales ciudades de Sudáfrica, o en tiendas instaladas en los townships, construidas en contenedores, denominadas spaza shops. En ellas, es posible conseguir a precios bajos productos de abarrotes, aunque con frecuencia estos son de baja calidad.

La combinación de tendencias que van de lo tradicional a lo novedoso brinda la posibilidad de observar cómo los migrantes que llegan al territorio sudafricano se adaptan a su nuevo entorno, y cómo entran en relación con otros actores como el Estado, las instituciones religiosas y las organizaciones no gubernamentales (ONG). Todos ellos buscan ya sea facilitar o dificultar la estancia de los migrantes en el territorio sudafricano.

Para entender mejor el abanico de posibilidades que resulta de esta situación, es importante analizar casos que ilustren las tendencias divergentes. Los siguientes apartados se enfocan en dos ejemplos de las formas en que la frontera se convierte en un espacio en disputa, donde diversos actores buscan maximizar sus intereses. Se estudia el caso de los migrantes que trabajan en las granjas del área de Musina, Limpopo, así como la actividad de los comerciantes transfronterizos.

\section{LA FRONTERA COMO LÍMITE A LOS DERECHOS DE LOS MIGRANTES}

Musina es un pueblo localizado en la frontera norte de Limpopo, aproximadamente a 20 $\mathrm{km}$ de Zimbabwe. Esta área recibe migrantes provenientes de Somalia, Etiopía y Malawi, aunque los migrantes zimbabwenses son los 
más numerosos debido a la proximidad geográfica y las condiciones socioeconómicas de su país de origen (отм, 2009).

La región constituye un punto de tránsito o de llegada. De tránsito, para quienes se dirigen a los centro urbanos como Johannesburgo, Ciudad del Cabo y Pretoria. De llegada, para los migrantes que deciden quedarse en la frontera, a fin de conseguir empleos temporales en un sitio cercano a Zimbabwe. De esta manera pueden adquirir alimentos, enviar dinero a sus familias o bien realizar el pago de las colegiaturas de sus hijos o familiares (огм, 2009), pero manteniéndose en una zona cercana a su lugar de origen

Entre los migrantes que deciden quedarse en Musina se encuentran personas que no cuentan con documentos oficiales ni con recursos para llegar a las grandes ciudades. Algunos, de hecho, prefieren permanecer en la zona (incluso cuando ganan salarios más bajos) como una estrategia para cruzar fácilmente la frontera y salir de Sudáfrica en caso necesario (Jinnah, 2017).

Muchas de estas personas laboran en granjas productoras de cítricos, melones, tomates, elote, calabaza, entre otros. Estos trabajos son estacionales. Además, existe otra categoría de migrantes: quienes tienen una actividad permanente debido a que cuentan con permisos de asilo o de visita de 90 días, o bien, fueron contratados bajo el esquema de los permisos corporativos que otorga el Department of Home Affairs (DHA) y que permiten a los dueños de las minas y las granjas emplear un número predeterminado de trabajadores durante un tiempo establecido (Jinnah, 2012).

La separación entre estas distintas categorías de migrantes muestra de manera clara la forma en que la frontera establece una distinción entre aquellas personas a quienes se le reconocen derechos y aquellas a quienes no se les reconocen, lo que se convierte en un elemento de control desde el punto de vista social y económico.

Al igual que en muchas partes del mundo, los trabajadores migratorios, especialmente quienes no cuentan con permisos de trabajo, sufren una fuerte explotación laboral. Por una parte, perciben salarios comparativamente bajos. Cada sector productivo cuenta con un sueldo establecido que se puede calcular con base en las horas trabajadas, el puesto desempeñado e incluso, por zonas geográficas (Department of Labour, s. f.). Los trabajadores migratorios sin documentos generalmente reciben pagos por debajo de los sueldos establecidos por sector productivo. Por ejemplo, una trabajadora doméstica que labore más de 27 horas a la semana en la zona A (Ciudad del Cabo, Johannesburgo y Pretoria), recibe un sueldo calculado de 2.545,22 rands ${ }^{2}$ al mes (MyWage.co.za., s. f.). Sin embargo, las trabajadoras extranjeras pueden recibir un pago menor si se encuentran en una situación migratoria irregular.

Algo similar sucede con los trabajadores agrícolas, quienes además enfrentan descuentos en sus sueldos por conceptos como pagos por agua, luz o pérdida de ganado. Algunos deben incluso comprar su propio equipo de pro-

2 Un dólar americano equivale a 12,48 rands. 
tección para el trabajo, como zapatos especiales para rociar productos químicos (Jinnah, 2012). Por otro lado, los trabajadores migratorios no reciben remuneración por horas extras, aunque sus jornadas laborales con frecuencia son de 11 horas, pues comienzan a las 6:00 y terminan a las 17:00 horas. Además, tampoco tienen vacaciones pagadas. El panorama se refleja en una frase ampliamente utilizada entre estos trabajadores: "No trabajo, no hay pago" ( p. 35).

En general, las malas condiciones casi nunca se reportan, porque los trabajadores temen sufrir represalias por parte de los granjeros o ser deportados. La situación se complica porque los migrantes enfrentan una gran falta de información sobre las instituciones u organizaciones que pueden ayudarlos en estos casos. Además, no cuentan con facilidades de transporte público para trasladarse desde las granjas hasta Musina y en sentido contrario.

Por estos motivos, otra idea muy extendida entre los migrantes es "estar debajo del radar": mantenerse invisibles es más seguro (Jinnah, 2012, p. 50). De esta manera, la situación de los trabajadores migratorios en Sudáfrica, especialmente de quienes han cruzado la frontera sin autorización oficial, ilustra las consideraciones de Aquino y Varela (2012) sobre el uso de la frontera y el derecho a cruzarla como un elemento de control por parte del Estado, que lo utiliza para precarizar un tipo muy específico de mano de obra.

\section{NEGOCIAR ESPACIOS DE OPORTUNIDAD: EL COMERCIO TRANSFRONTERIZO}

Otro espacio importante en donde se refleja el papel múltiple y disputado de las fron- teras es en la actividad comercial, sobre todo de pequeña escala. El comercio transfronterizo ha registrado una rápida expansión en las últimas décadas. La actividad se concentra en personas procedentes de los países vecinos de Sudáfrica aunque, como se señaló, también es común encontrar ciudadanos de países africanos más lejanos. Todos ellos ingresan al territorio sudafricano para adquirir mercancías que luego llevan a sus lugares de origen para venderlas entre familiares y amigos, o en distintos tipos de establecimientos, como restaurantes o tiendas, ya sea en pueblos o ciudades.

Se desconoce el número exacto de migrantes dedicados al comercio transfronterizo debido a que no existen estadísticas oficiales al respecto (Peberdy et al., 2015a). No obstante, hay algunos indicadores que señalan claramente la importancia de esta actividad. Un promedio de cerca de 465 autobuses sale diariamente de la ciudad sudafricana de Johannesburgo en dirección a los países vecinos: Botswana, Malawi, Namibia, Mozambique, Zambia y Zimbabwe (Stephen, 2017). De acuerdo con Zack (2017), la mayoría de los visitantes que llegan a Sudáfrica a través de los puestos fronterizos (es decir, por tierra y no por aire), son comerciantes de países vecinos. Si ambos datos se entrelazan, se puede concluir que el comercio transfronterizo en Sudáfrica es muy activo.

En la actualidad, el comercio transfronterizo se ha convertido en un modo de vida para muchas personas, tanto en Sudáfrica como en los países vecinos. De hecho, en muchos hogares constituye la actividad económica primaria, o incluso la única (Chikanda y Tawodzera, 2017). Estimaciones informales han señalado que un comprador puede gastar un promedio de 14.254 rands en un día, y que el total de las 
operaciones puede ascender anualmente a 10 mil millones de rands (Zack, 2017).

Algunos comerciantes compran productos en los mercados informales de sus países de origen (Zimbabwe, Mozambique, Malawi, Botswana, Zambia, Angola, Namibia), para luego venderlos en Sudáfrica. Las mercancías introducidas a Sudáfrica incluyen alimentos (anacardo, pan, pescado), textiles (servilletas, capulanas, ropa tradicional), tejidos, así como artesanías (canastas, tambores, entre otros) (Peberdy, 2015b).

Por otro lado, muchos llegan a Sudáfrica para adquirir una amplia variedad de bienes que luego comercializarán en sus lugares de origen. Entre los artículos más solicitados se cuentan alimentos, ropa, zapatos, mantas, juguetes, enseres domésticos, teléfonos celulares, aparatos electrónicos, refacciones automotrices, materiales de construcción, cigarros, alcohol, entre otros. Algunos recurren a internet y las redes sociales para acercarse a los posibles compradores: "utilizan WhatsApp o Facebook para subir fotografías de, por ejemplo, un bolso rosa o unos tenis, a fin de que sus clientes los vean y ordenen" (Stephen, 2017).

Todos estos productos se adquieren tanto en tiendas establecidas como en centros comerciales informales que venden productos extranjeros, sobre todo chinos, como el China Mall. Los principales puntos de abastecimiento son Johannesburgo y Pretoria, aunque existen otras zonas donde los comerciantes adquieren sus mercancías (Raimundo y Chikanda, 2016). Los mozambiqueños, por ejemplo, con frecuencia compran en pueblos cercanos a la frontera entre Mozambique y Sudáfrica, como Nelspruit o Malelane, e incluso en Swazilandia.
Los comerciantes transfronterizos no suelen permanecer en territorio sudafricano por periodos largos. La duración de su estancia dependerá sobre todo de dos factores: si únicamente entraron para comprar o si tienen mercancías para vender, y si pueden afrontar los altos costos de hospedaje y otros factores más como el temor a que sus productos sean robados (Peberdy et al., 2015).

La actividad comercial tan dinámica, $y$ que involucra a actores tan diversos, ha llevado a la formación de complejas redes que en distintos sentidos desafían la territorialidad establecida por las fronteras y se convierten en un espacio de resistencia contra el Estado.

Los comerciantes transportan los productos sobre todo en autobuses y minibuses públicos. Generalmente, pagan sus impuestos al llegar a la frontera, pero existen prácticas alternativas para no hacerlo, como solicitar a un particular que lleve en su equipaje una parte de las mercancías (Peberdy et al., 2015a).

Raimundo y Chikanda (2016) señalan que las prácticas irregulares no solo están a cargo de los comerciantes, sino que también las pueden realizar los empleados gubernamentales. Algunas personas dedicadas al comercio transfronterizo indican que existe corrupción en las aduanas, donde los oficiales piden sobornos a cambio de dejar pasar las mercancías sin complicaciones. Por ejemplo, un comerciante que quiera cruzar la frontera con 20 cajas de fruta, puede pagar una cantidad al oficial que revisa su carga y después pagar en la aduana el impuesto correspondiente a solo 10 cajas.

De esta manera, las fronteras se convierten en espacios donde tanto el Estado como los comerciantes buscarán formas de operar 
en su propio beneficio: el primero tratando de cobrar impuestos sobre las mercancías o a través de la corrupción, y los segundos desplegando estrategias para evitar o reducir el pago de esos impuestos, minimizar los efectos de la corrupción y maximizar las posibles ganancias.

La continua discrepancia que se presenta en las fronteras entre los particulares y el Estado se refleja en otro ámbito: los comerciantes han reportado casos de abusos verbales por parte de la policía, el ejército y los oficiales fronterizos. Incluso, algunos afirmaron haber sido víctimas de violencia física en ambos lados de la frontera (Raimundo y Chikanda, 2016).

Además, muchos enfrentan también problemas de seguridad al volver a sus países de origen. En el caso de Mozambique, los comerciantes han reportado ataques en los minibuses que los transportaban, por parte de bandidos que colocan esteras con clavos para pinchar las llantas (Raimundo y Chikanda, 2016).

\section{EL MARCO LEGAL: ALCANCES Y LIMITACIONES}

Como se observa, la movilidad en las fronteras hacia Sudáfrica implica importantes retos para quienes las cruzan. Muchos de ellos se enfrentan, en diferentes grados, a situaciones de violencia, inseguridad, incertidumbre y explotación. Ante ello, resulta conveniente analizar el marco jurídico sudafricano relacionado con los migrantes y las fronteras.

En cuanto al comercio transfronterizo, Rogerson (2015) detectó que el gobierno pos-apartheid comenzó a adoptar una política de apoyo a la economía informal a partir de 2004; sin embargo, dicho apoyo estaba condicionado por la nacionalidad y el estatus migratorio de las personas involucradas. Los refugiados y otros tipos de migrantes no son beneficiarios de los programas gubernamentales. Específicamente, la Licensing of Businesses Bill de 2003 establece como requisito para poder dedicarse a la venta de productos que la estancia en el país sea legal y el interesado cuente con un permiso para comerciar, en los términos de la Immigration Act o la Refugee Act. Dichas disposiciones excluyen la posibilidad de que aquellos migrantes en situación irregular puedan regularizar su situación legal fácilmente. Frente a este panorama, Peberdy (2016) considera que el Gobierno debe tratar de incorporar los negocios de los migrantes, en lugar de excluirlos y catalogarlos como algo negativo, pues es necesario que se reconozca su contribución a la economía sudafricana y se les deje de estigmatizar con la ilegalidad.

Respecto de los trabajadores migratorios existen diversos instrumentos legales que regulan su situación. Entre ellos se cuenta la Refugee Act 130 de 1998 y su Amendment 33 de 2008, donde se establece que los refugiados tienen todo tipo de derechos excepto a votar en territorio sudafricano; por su parte, la Basic Conditions of Employment 75 de 1997, y la Sectoral Determination 13 for Farm Workers disponen el pago de un sueldo básico establecido para dicho sector productivo y un límite máximo de horas que estas personas pueden trabajar; finalmente, la Occupational Health and Safety Act 85 de 1993 señala que los empleadores deben garantizar la salud y seguridad de todos los trabajadores, sin importar su nacionalidad o estatus migratorio (Jinnah, 2012). 
A pesar de la existencia de una legislación tan sensible a las necesidades de los migrantes, Ramjathan-Keogh (2017) advierte sobre la posibilidad de que el derecho al trabajo para los refugiados se vea limitado, de ser aceptada una enmienda que se ha propuesto para la sección 15 de la Refugee Act, la cual establece que quienes puedan sostenerse económicamente no podrán trabajar por un periodo de cuatro meses. Por otro lado, aquellos que no cuenten con medios económicos podrán recibir ayuda del Alto Comisionado de las Naciones Unidas para los Refugiados (ACNUR) pero se les negará el derecho a trabajar. De concretarse, estos cambios implicarían un retroceso, puesto que de acuerdo con la actual legislación los refugiados pueden buscar trabajo desde el momento en que se internan en el territorio sudafricano.

Aunado al marco jurídico nacional, los instrumentos internacionales constituyen otro pilar fundamental para la protección de los migrantes. Entre los instrumentos internacionales más relevantes en la materia a los cuales se ha adherido Sudáfrica se cuenta la oua Convention Governing the Specific Aspect of Refugee Problems in Africa, promulgada en 1969, y que se mantiene hasta la actualidad como el instrumento jurídico fundamental para la protección de los refugiados en el continente.

Con el reemplazo de la Organización para la Unidad Africana (OUA) por la Unión Africana (UA), los países africanos buscaron consolidar una posición común frente a la migración, a través de dos documentos adoptados en el 2006: la African Common Position on Migration and Development y la Migration Policy Framework for Africa. Ambas proporcionan una orientación detallada sobre la forma co- mo los Estados africanos deberían regular la migración. Sin embargo, ninguna de ellas es vinculante para los Estados miembros (Landau y Achiume, 2015).

Existen otros instrumentos legales relacionados con los migrantes a los que Sudáfrica no se ha adherido. Tal es el caso de la Convention on All Migrant Workers and their Families de 1990 (United Nations Treaty Collection), documento elaborado por la Organización de las Naciones Unidas (ONU). La firma de esta convención por parte de Sudáfrica constituiría un gran avance en materia de derechos de los trabajadores migratorios, pues con ella se busca garantizar un trato igualitario para trabajadores tanto migrantes como nacionales. El instrumento reconoce los derechos de los migrantes legales, pero señala que incluso los trabajadores migratorios en situación irregular deben gozar del respeto de sus derechos humanos (UNESCO, 2005).

Así, en el caso de los trabajadores migratorios, al igual que con los comerciantes transfronterizos, existe una constante tensión frente al Estado y sus intereses. En la vida cotidiana de los migrantes se imponen limitantes a causa de las iniciativas gubernamentales o por la falta de cumplimiento de las leyes establecidas. Este último caso se puede observar, por ejemplo, en el incumplimiento del límite máximo de horas de trabajo establecidas por la ley. Si bien en este caso quienes no acatan la normatividad son particulares (los dueños de las granjas), el Estado no obliga al cumplimiento de la ley. Por una parte, existen limitantes a través de la normatividad misma, pues para entrar en las granjas se requiere normalmente del permiso de los dueños, lo cual representa 
un obstáculo para el monitoreo. Por otro lado, en ocasiones los agentes gubernamentales contribuyen de manera activa, como se observa en las acusaciones de choque entre la policía y los empleadores. En ocasiones, al final de mes los dueños de las granjas reportan ante la policía a los trabajadores migratorios que no cuentan con documentación, a fin de no pagarles los sueldos correspondientes (Jinnah, 2017).

Además del ámbito laboral, los migrantes enfrentan problemas también en el acceso a servicios médicos. Ponthieu e Incerti (2016) señalan que mientras se desplazan, los migrantes corren diversos peligros físicos como violaciones y detenciones, e incluso, la adquisición de enfermedades a causa de las malas condiciones de sanidad.

Después de su etapa de tránsito, una vez se han establecido en algún lugar, los migrantes siguen teniendo problemas en lo relativo al acceso a la salud. Un ejemplo se encuentra en el ya citado caso de Musina. Las granjas se encuentran en áreas aisladas y lejanas. En toda la región solo hay una autopista, que une a la ciudad de Pretoria con la frontera con Zimbabwe; existen además dos carreteras asfaltadas. Fuera de eso, solo hay caminos sin asfaltar. Además, el transporte público es muy escaso, de modo que para salir de las granjas los trabajadores deben caminar largas distancias (Jinnah, 2017). Debido a esta situación, el acceso a la asistencia médica es difícil. Muchos migrantes dependen directamente de los servicios itinerantes proporcionados a través de clínicas móviles (orm, 2009; Médicos sin Fronteras, 2013). Inicialmente, este tipo de servicios era ofrecido por la Iом y por Médicos sin Fronteras. Para los migrantes en situación irregular, este servicio ofrecía una percepción de mayor seguridad, porque al utilizarlo evitaban el riesgo de ser arrestados en los hospitales públicos. Sin embargo, en 2013 Médicos sin Fronteras dejó de operar las clínicas móviles y trasladó su manejo al Department of Health y a socios no gubernamentales (Médicos sin Fronteras, 2013).

Otro espacio problemático es el de la asistencia legal. Como en el caso de los servicios médicos, el acceso de los migrantes a este tipo de servicios es limitado. De hecho, muchos prefieren no buscar apoyo legal ni siquiera en los casos en que sus derechos han sido violentados, como parte de la estrategia de "permanecer bajo el radar" para no llamar la atención de las autoridades sudafricanas (Jinnah, 2017).

Por otro lado, las ONG que brindan asistencia a los migrantes, como la Mamedi Legal Advice Office, señalan que su tarea se dificulta por la lejanía geográfica de las granjas y por la necesidad de contar con un permiso previo, que generalmente deben otorgar los dueños de cualquier granja, para poder entrar a su propiedad. En ciertos casos, existe la posibilidad de realizar inspecciones de manera conjunta con las ONG, con un permiso facilitado por el Department of Labour, pero esta instancia gubernamental cuenta con un número limitado de inspectores laborales y vehículos (Jinnah, 2012), lo cual dificulta su labor.

\section{EL GOBIERNO SUDAFRICANO Y EL ASEGURAMIENTO DE LAS FRONTERAS}

Sin lugar a dudas, los elementos legales relacionados con la presencia de los migrantes 
en Sudáfrica constituyen una piedra angular para comprender la actuación del Gobierno sudafricano en la materia pero, como se ha podido observar, existen otros elementos que entran en juego para entender la dinámica que se establece en las fronteras. Los temas de la vigilancia y la seguridad, configurados de manera significativa por ciertas coyunturas específicas, han sido una preocupación constante para Sudáfrica.

En el periodo del apartheid, sobre todo durante la década de los ochenta, la creciente efervescencia política y social entre una población cansada del dominio por parte de una minoría y la represión ejercida por el Gobierno tuvo un impacto en el manejo de las fronteras.

McMichael (2012) y Mahlangu (2016) explican que durante ese periodo el régimen buscó garantizar la seguridad fronteriza y contener posibles ataques por parte de movimientos de liberación, recurriendo a métodos como la colocación de rejas eléctricas en las fronteras y el patrullaje regular por parte de la policía, las unidades civiles auxiliares y el ejército. Se registró así un fuerte despliegue militar. En los límites con la actual Namibia (entonces denominada South West Africa, territorio que Sudáfrica mantuvo por la fuerza bajo su control hasta 1990), la presencia militar tenía por objetivo debilitar al movimiento independentista conocido como South West African People's Organisation (swapo). En la frontera con Angola se buscaba igualmente desestabilizar a la SWAPO, pero también al grupo denominado Umkhonto we Sizwe (MK), brazo armado del African National Congress (ANC). Este también constituía el motivo de una fuerte preocupación gubernamental en la frontera con Mozambique.
Los gobiernos pos-apartheid trataron de reducir la presencia militar en las áreas fronterizas. De manera especial, durante el periodo presidencial de Thabo Mbeki (1999-2008) se intentó reducir la actividad del ejército y aumentar progresivamente la participación del South African Police Service (saps) (McMichael, 2012).

No obstante, la tendencia se revirtió con la celebración de la Copa Mundial de Fútbol 2010 en suelo sudafricano. En ese momento, el gobierno de Jacob Zuma volvió a otorgar al ejército un papel preponderante en la vigilancia de las fronteras. Paradójicamente, la renovada importancia del ejército contrastaba con el limitado presupuesto que se le asignó; esto ocasionó que el regreso de los militares a las fronteras se diera por etapas. Por ejemplo, la South African National Defence Force (SANDF) volvió a las fronteras de Mozambique, Zimbabwe y Botswana tan solo en abril del 2011 (Global Security, s. f.).

Hasta el momento, la SANDF se mantiene en las fronteras en un esfuerzo por garantizar la seguridad contra amenazas como el tráfico ilegal de drogas, armas, vehículos, ganado e incluso personas. Para ello, ha incorporado nuevas tácticas y ha emprendido la modernización de su equipo, por ejemplo, de los vehículos que utiliza (Holder, 2017; Gwangwa, 2017).

El regreso de la SANDF a las zonas fronterizas no significa que el SAPS ya no participe en la vigilancia. Sudáfrica mantiene una política de cooperación interinstitucional a través de la cual varios organismos estatales intervienen en el tema de la seguridad y el control de las fronteras. La policía se enfoca en la protección de las instalaciones fronterizas y en el combate a delitos cometidos en las fronteras, así como 
a la vigilancia de personas y mercancías en puertos de entrada. Además, realiza labores de inteligencia, entre otras actividades (Mahlangu, 2016).

A pesar de la cooperación interinstitucional entre la policía y el ejército, la vigilancia de las fronteras presenta serias dificultades puesto que ambas instituciones tienen presupuesto y personal limitados. La situación se vuelve más problemática debido a que la extensión de las fronteras terrestres y sus condiciones geográficas dificultan la labor de las fuerzas gubernamentales.

Estos problemas se traducen en situaciones de inseguridad para muchos migrantes. Específicamente, en el caso de los migrantes irregulares que cruzan por las áreas cercanas al río Limpopo, se han reportado numerosos casos de violencia, ya que atraviesan por zonas aisladas dominadas por delincuentes (que actúan con frecuencia en bandas conocidas como magumaguma). En algunas ocasiones, la violencia puede provenir también de los soldados que están a cargo de la seguridad del área.

Existen grupos especialmente vulnerables como las mujeres, quienes se arriesgan a sufrir tanto robos como ataques sexuales (Ponthieu e Incerti, 2016; Bolt, 2017). Para ellas, la violencia puede prolongarse incluso cuando han conseguido un empleo en las granjas fronterizas. Bolt (2017) sostiene que el riesgo se genera en parte porque la violencia se convierte en una forma en que los trabajadores tratan de expresar autoridad en un contexto en que en que su poder y capacidad de acción se ven continuamente limitados. Infortunadamente, es frecuente que las víctimas de violación no denuncien los hechos por temor a ser deportadas (oIM, 2009).

\section{OTROS ACTORES EN LAS FRONTERAS}

Además del Estado y quienes cruzan la frontera, que se ubican en cierta forma en polos opuestos del espectro, existen otros actores que buscan tener una mayor incidencia en la construcción de los espacios fronterizos.

Hlatshwatyo (2016) subraya que existen ONG enfocadas en la ayuda a los migrantes. La mayoría concentra sus esfuerzos en los principales centros urbanos de Sudáfrica, como Pretoria, Johannesburgo, Durban y Ciudad del Cabo. Sin embargo, el campo de acción de dichas organizaciones también cubre las fronteras (Rutherford, 2012). Por ejemplo, en el caso de Musina, se registró un incremento en la participación de ONG en la zona, especialmente entre 2004 y 2009, cuando el tránsito de migrantes se incrementó de manera notable debido al aumento de zimbabwenses que huían de la crisis económica y política imperante en su país.

Entre las organizaciones no gubernamentales que tienen presencia en las fronteras sudafricanas se cuentan algunas iglesias como la Uniting Reform Church, y organizaciones como Médicos sin Fronteras o la Organización Internacional para las Migraciones (OIM). Dichos actores han adquirido un papel relevante debido a que proveen abrigo, comida y servicios de salud a la población migrante.

Algunos establecimientos brindan albergue a quienes cruzan la frontera para internarse en Sudáfrica. Específicamente, la Uniting Reform Church cuenta con dos albergues dedicados a niños, jóvenes y mujeres, en tanto que la Iglesia católica cuenta con un albergue para mujeres que buscan asilo, provenientes principalmente de Zimbabwe y el Congo. Sin embargo, estos centros no son suficientes 
ante la gran cantidad que personas que diariamente realizan el cruce de la frontera. La mayoría de las personas que llegan tiene que limitar su estancia a tres días, aunque en casos excepcionales, como migrantes enfermas o que hayan dado a luz recientemente, este periodo se puede extender (sacbC AIDs Office News, s. f.). Rutherford (2012) afirma que en algunos de estos centros religiosos los migrantes consiguen trabajos temporales desempeñando labores como la limpieza de terrenos, previa aprobación de las autoridades religiosas y con el apoyo de miembros de las iglesias.

Además de ofrecer albergue, la Iglesia católica distribuye paquetes de comida a los migrantes. La demanda creció de manera particular en la segunda mitad de la década del 2000 (Rondganger, 2008), con el aumento de la migración proveniente de Zimbabwe a causa de la violencia política y la catástrofe económica del país. Resta por ver el impacto que pueda tener en el fenómeno migratorio la llegada de un nuevo presidente al Gobierno de Zimbabwe, en sustitución de Robert Mugabe, ocurrida en 2017. En todo caso, el efecto del cambio de régimen no ha sido inmediato, pues Zimbabwe debe recorrer un largo camino hacia la reconciliación política y la reactivación económica.

Por el momento, la demanda de albergue para los migrantes sigue superando la oferta existente. Como resultado, son frecuentes los casos de personas que deben quedarse a campo abierto, sin ningún tipo de servicios básicos. Indudablemente, esto tiene implicaciones sanitarias. Un ejemplo de los efectos negativos de esta situación ocurrió en 2008, cuando se registró un brote de cólera traído por migrantes zimbabwenses en búsqueda de ayuda médica (Rondganger, 2008). En 2018, las preocupaciones resurgieron a causa de un nuevo brote de la misma enfermedad, que afectó a Zimbabwe así como a algunos países vecinos, especialmente Mozambique y el norte de Sudáfrica (Thompson, 2018).

Ante ese panorama, el manejo de la situación sanitaria en las fronteras se vuelve imperativo. Por esta razón, el tema de la salud de los migrantes constituye una preocupación central de algunas organizaciones como Médicos sin Fronteras y la oim. Así, se observa que los actores no estatales están tratando de resolver una necesidad ante la falta de respuesta del Estado, como ha ocurrido en otros ámbitos.

\section{CONCLUSIÓN}

El caso sudafricano ilustra con claridad la existencia de las fronteras como elementos de múltiples significados y en constante construcción. La confluencia de una variedad de actores con capacidades e intereses distintos hace imposible concebir estos espacios como realidades fijas y con sentidos determinados de antemano.

Las fronteras de Sudáfrica aparecen de manera alternativa como espacios problemáticos o como zonas de oportunidad, dependiendo de las condiciones socioeconómicas y políticas imperantes en la propia Sudáfrica y en los países de África Austral. Puesto que estas condiciones no son estáticas, sino que se transforman a lo largo del tiempo, las realidades en las fronteras se modifican también.

De esta forma, lo que en un momento puede constituir un obstáculo, en otros casos 
puede funcionar como una ventaja. Así ocurre en el caso de los trabajadores migratorios en las granjas cercanas a la frontera. Por una parte, es común que sufran abusos y el trabajo que realizan suele ser mal remunerado; esto sin duda es un problema. No obstante, factores como la cercanía con su país de origen y las facilidades que tienen en la práctica para atravesar las porosas fronteras sudafricanas son considerados por muchos migrantes como una ventaja que ellos están dispuestos a aprovechar tanto como sea posible.

Por su parte, quienes se dedican al comercio transfronterizo enfrentan igualmente una serie de desafíos relacionados con su seguridad personal y la de sus mercancías, la corrupción de las autoridades y las dificultades inherentes a su ocupación. Al mismo tiempo, el comercio a través de las fronteras se ha convertido en una opción atractiva para muchas personas, lo cual se refleja en el volumen de las operaciones.

En ambos casos, el significado de la frontera no puede establecerse de manera definitiva, sino que se construye de forma contextual. Con frecuencia, los análisis más tradicionales de las fronteras como unidades fijas pueden pasar por alto estos matices. En cambio, si se interpreta la realidad desde perspectivas alternas que consideren múltiples niveles, se alcanzarán más elementos para la comprensión de las realidades cambiantes.

Solo de esta forma podemos entender a las fronteras como elementos que separan, pero que al mismo tiempo constituyen puntos de contacto. Ciertos actores pueden privilegiar una de estas facetas, mientras que otros pueden enfatizar la cara contraria de la moneda. Sin embargo, ambos aspectos conforman una totalidad que se encuentra en constante movimiento.

En el caso de Sudáfrica, es frecuente que el Estado subraye la existencia de las fronteras como elemento de separación. De manera a veces explícita y a veces velada, a menudo las autoridades centran sus esfuerzos en resaltar la existencia de un "yo" sudafricano que se distinguirá claramente del "otro" extranjero, al cual muchas narrativas han clasificado como una fuente constante de peligro. Esto puede ser aprovechado incluso por actores no estatales, como los dueños de granjas que utilizan la mano de obra migratoria, o por aquellos sudafricanos que se sienten afectados por la presencia del migrante, como los trabajadores locales que los ven como competencia. Por su parte, quienes cruzan las fronteras para buscar mejores condiciones de vida pueden resaltar, al menos de manera implícita, el papel de las fronteras como puntos de contacto. De esta manera se establece un continuo proceso de negociación de significados.

\section{REFERENCIAS}

African Union (2017). List of Countries which have signed or ratified to the African Union Convention for the Protection and Assistance of Internally Displaced Persons in Africa (Kampala Convention). Recuperado de https://au.int/sites/ default/files/treaties/7796-sl-african_union_ convention_for_the_protection_and_assistance_of_internally.pdf

Aquino. A y Varela. A. (2012). Introducción. Pensar la migración en el contexto capitalista actual. En Aquino y Decossé (eds.), Desafiando fronteras. 
Control de la movilidady experiencias migratorias en el contexto capitalista. Oaxaca: Frontera Press.

Beurskens, K. y Miggelbrink, J. (2017). Special section introduction - Sovereignty contested: Theory and practice in borderlands. Geopolitics, 22 (4), 749-756.

Bolt, M. (2016). Mediated paternalism and violent incorporation: Enforcing farm hierarchies on the Zimbabwean-South African border. Journal of Southern African Studies, 42(5), 911-927.

Brunet-Jailly, E. (2005). Theorizing borders: An interdisciplinary perspective. Geopolitics, 10,633-649.

Chikanda, A. y Tawodzera, G. (2017). Informal Entrepreneurship and Cross-Border Trade between Zimbabwe and South Africa. Cape Town: Southern African Migration Programme (sAmP).

Crush, J., Chikanda, A. y Skinner, C. (2015). Migrant Entrepreneurship and Informality in the South African Cities. En J. Crush, A. Chikanda y C. Skinner (eds.). Mean Streets. Migration, Xenophobia and Informality in South Africa (pp. 1-24). Cape Town: Southern African Migration Programme.

Department of Labour (s. f.). Sectoral Determination 7: Domestics Workers. Recuperado de http:// www.labour.gov.za/DoL/legislation/sectoraldeterminations/sectoral-determination-7-domestic-workers

Foucher, M. (1991). Fronts et frontières. Un tour $d u$ monde géopolitique. Paris: Fayard.

Global Security (s. f.). Borderline Policing. Recuperado de https://www.globalsecurity.org/military/world/rsa/ borderline-policing.htm

Gwangwa, V. (2017). SANDF in border crime drive. IOL. Recuperado de https://www.iol.co.za/ pretoria-news/news/sandf-in-border-crimedrive-12046670.
Hlatshwatyo, M. (2016). Xenophobia, resilience, and resistance of immigrant workers in South Africa: Collective and individual responses. En A. Choudry y Mondli, H. (eds.). Just Work? Migrant Worker's Struggles Today (pp. 21-40). London: Pluto Press.

Holder, T. (2017). The SANDF dominates sA borders. Eyewitness News. Recuperado de http://ewn. co.za/2017/11/17/watch-sandf-dominates-saborders).

Jinnah, Z. (2012). "We Have to go into the Bush." Understanding the responses of $N G O$ and government in addressing conditions faced by crossborder migrant workers in Musina. Case Study: Musina, Limpopo, South Africa. Johannesburgo: University of Witwatersrand-Hivos.

Jinnah, Z. (2017). Silence and invisibility: Exploring labour strategies of zimbabwean farmworkers in Musina, South Africa. South African Review of Sociology, 48 (3), pp. 46-63.

Khumalo. T. (2016). S. Africa Urged to Stop Abuse of Migrant Farm Workers. Voanews. Voanews. Recuperado en https://www.voanews.com/a/southafrica-migrant-farm-workers/3154755.html

Kimambo, I. N. (1997). The interior before 1800. En I. N. Kimambo y A. J. Temu (eds.). A history of Tanzania (pp. 14-33). Dar es Salaam: Kapsel Educational Publications.

Landau, L. y Tendayi Achiume, E. (2015). The African Union Migration and regional integration framework. Recuperado de http://www.accord. org.za/publication/the-african-union-migrationand-regional-integration-framework/

Ley, D. (2005). Shaky Borders? Transnational migrants as strategic actors. En H., van Houtum, O. Kramsch y W. Zierhofer (eds.). B/ordering space (pp. 61-75). Burlington: Ashgate. 
Mahlangu, S. B. (2016). Effectiveness and challenges of South African Police Service and Security Agencies on land border security and control. Thesis for the degree Doctor Technologiae in Policing at Tshwane University of Technology. Recuperado de

http://tutvital.tut.ac.za:8080/vital/access/manager/ Repository/tut:2334

McMichael, C. (2012). The remilitarisation of South Africa's borders. Recuperado de https://www. opendemocracy.net/chris-mcmichael/re-militarisation-of-south-africa $\% \mathrm{E} 2 \% 80 \% 99$ s-borders

Médicos sin Fronteras (2013). Mobile care for mobile populations: MSF leaves Musina, South Africa, with a legacy of innovation, 02 de diciembre de 2013. Recuperado de https://www.msf.org.uk/ article/mobile-care-mobile-populations-msfleaves-musina-south-africa-legacy-innovation

Minghi, J. V. (1991). From conflict to harmony in border landscapes. En D. Rumley y J. V. Minghi (eds.). The geography of border landscapes (pp. 15-30). London and New York: Routledge.

Mywage. Minimum Wages in South Africa. Recuperado de https://mywage.co.za/salary/minimumwages/

Newman, D. y Paasi, A. (1998). Fences and neighbours in the postmodern world: Boundary narratives in political geography. Progress in human. Geography, 22 (2), pp. 186-207.

Oxford University Press (2008). The implementation of Apartheid. En Understanding Apartheid. Apartheid Museum. Recuperado de http://www. apartheidmuseum.org/sites/default/files/files/ downloads/Learners\%20book\%20Chapter3.pdf

Paasi, A. (1998). Boundaries as social processes: Territoriality in the world of flows. Geopolitics, 3 (1), pp. 69-88.
Paasi, A. (2005). The changing discourse on political boundaries. Mapping the backgrounds, contents and contexts. En H. van Houtum, O. Kramsch y W. Zierhofer (eds.). B/ordering space (pp. 1731). Burlington: Ashgate.

Parker y Vaughan-Williams et al. (2009). Lines in the Sand? Towards an Agenda for Critical Border Studies. Geopolitics, 14, 582-587.

Peberdy, A. S. (2010). Mobile entrepreneurship: Informal sector cross-border trade and street trade in South Africa. Development Southern Africa, 17 (2), 201-219

Peberdy, A. S. et al. (2015a). Calibrating Informal Cross-Border Trade in Southern Africa, Cape Town. Migration Policy Series 69. Recuperado de http://imrc.ca/wp-content/uploads/2015/05/ PS-69-Calibrating-Informal-Cross-Border-Trade-in-Southern-Africa.pdf

Peberdy, A. S. et al. (2015b). Transnational entrepreneurship and informal cross-border trade with South Africa. En J. Crush, A. Chikanda y C. Skinner (eds.). Mean Streets. Migration, Xenophobia and Informality in South Africa (pp. 207-228). Cape Town: Southern African Migration Programme.

Peberdy, A. S. (2016). International migrants in Johannesburg's informal economy. SAMP Migration Policy Series, 71. Recuperado de http:// www.gcro.ac.za/media/reports/SAMP71.pdf

Ponthieu, A. e Incerti, A (2016). Continuity of care for migrants populations in Southern Africa. Refugee Survey Quarterly. Oxford University Press.

Portes, A. (2013). Sociología económica: una investigación sistemática. Madrid: Centro de Investigaciones Sociológicas.

Pfoser, A. (2015). Between security and mobility: Negotiating a hardening border regime in the Russian-Estonian borderland. Journal of Ethnic and Migration Studies, 41 (10), 1684-1702. 
Raimundo, I. y Chikanda, A. (2016). Informal Entrepreneurship and Cross-Border Trade in Maputo, Mozambique. SAMP Migration Policy Series, 73. Recuperado de http://samponline.org/wp-content/uploads/2016/10/Acrobat73.pdf

Ramjathan-Keogh, K. (2017). The rights of refugees and migrant workers. En F. Veriava y A. Thom (eds.). Basic Education Rights Handbook. Education Rights in South Africa, Section 27. Recuperado de http://section27.org.za/wp-content/ uploads/2017/02/Chapter-6.pdf

Rioja, L. (2015). El imaginario de la seguridad en la frontera sur de México a principios del siglo xxi. Península, X (1), 29-47. Recuperado de https://ac.els-cdn.com/S1870576615000033/1s2.0-S1870576615000033-main.pdf? tid $=$ spdf $-7 \mathrm{c} 6 \mathrm{dc} 7 \mathrm{ed}-8347-487 \mathrm{c}-9 \mathrm{eb} 2$ cc4a82043469\&acdnat $=1519906176 \_$e300d d5d01573a333916803151eea84f

Rogerson, C. M. (2015). Unpacking national policy towards the urban informal economy. En J. Crush, A. Chikanda y C. Skinner (eds.), Mean Streets. Migration, Xenophobia and Informality in South Africa (pp. 229-249). Cape Town: SAMP.

Rondganger, L. (2008). Musina at breaking point as refugee pour in. Recuperado de https://www.iol. co.za/news/politics/musina-at-breaking-pointas-refugees-pour-in- 429590

Rumford, C. (2012). Towards a multiperspectival study of borders. Geopolitics, 17, pp. 887-902.

Rutherford, B. (2012). The uneasy ties of working and belonging: The changing situation for undocumented Zimbabwean migrants in Northern South Africa. En A. Bloch y M. Chimienti. Irregular Migrants. Policy. Politics, Motives and Everyday Lives. Oxon: Routledge.

Saunders, C. T. (1983). Historical dictionary of South Africa. Metuche, N. J.: Scarecrow.
SACBC AIDS Office News (2012). Women's refugee shelter, Musina, Diocese of Tzaneen. Recuperado de http://aidsoffice.sacbc.org.za/womens-refugeeshelter-musina-diocese-of-tzaneen/

Sthephen, J. (2017), Welcome to centre of Africa's shopping. Business Day, 06 de diciembre. Recuperado de https://www.businesslive.co.za/bd/ life/books/2017-12-06-book-review-welcometo-centre-of-africas-shopping/

South African History On Line (s. f.) Sophiatown. Recuperado de http://www.sahistory.org.za/ place/sophiatown

International Organization for Migration (2009). Migrants' Needs and Vulnerabilities in the Limpopo Province, Republic of South Africa. Pretoria: International Organization for Migration, Regional Office for Southern Africa Recuperado de https:// reliefweb.int/sites/reliefweb.int/files/resources/ EF29025533EE23B8C12575670042267Afull_report.pdf

Thompson, J. (2018) No handshakes at funerals as cholera spreads in Zimbabwe. TimesLive. Recuperado de https://www.timeslive.co.za/news/ africa/2018-02-02-no-handshakes-at-funeralsas-cholera-spreads-in-zimbabwel

UNESCO (2005). Information Kit. United Nations Convention on Migrant Rights. Recuperado de http://unesdoc.unesco.org/images/0014/0014 35/143557e.pdf

United Nations Treaty Collection (2018). International Convention on the Protection of the Rights of All Migrant Workers and Members of the Families. Recuperado de https://treaties.un.org/Pages/ ViewDetails.aspx?src=IND\&mtdsg_no $=I V-$ $13 \&$ chapter $=4 \&$ lang $=$ en

Vale, P. (2003). Security and politics in South Africa. The Regional Dimension. Boulder, London: Lynne Ryenner. 
Werlen, B. (2005). Regions an everyday regionalizations. From a space-centered towards an action-centered human geography. En H. van Houtum, O. Kramsch y W. Zierhofer (eds.). B/ordering space (pp. 47-60). Burlington: Ashgate.

Zack, T. (2017). Cross Border Shopping in Johannesburg's

Inner City. Johannesburgo: Johannesburg Inner City Partnership.
Zeller, W. (2009). Danger and opportunity in Katima Mulilo: A namibian border boomtown at transnational crossroads. Journal of Southern African Studies, 35 (1), 133-154. 